\title{
A linear boundary value problem for weakly quasiregular mappings in space
}

\author{
Baisheng Yan \\ Department of Mathematics, Michigan State University, East Lansing, MI 48824, USA \\ (e-mail: yan@math.msu.edu)
}

Received July 20, 2000 / Accepted September 22, 2000 /

Published online December 8, 2000 - (c) Springer-Verlag 2000

\begin{abstract}
Given a number $L \geq 1$, a weakly $L$-quasiregular map on a domain $\Omega$ in space $\mathbf{R}^{n}$ is a map $u$ in a Sobolev space $W_{l o c}^{1, p}\left(\Omega ; \mathbf{R}^{n}\right)$ that satisfies $|D u(x)|^{n} \leq L \operatorname{det} D u(x)$ almost everywhere in $\Omega$. In this paper, we study the problem concerning linear boundary values of weakly $L$-quasiregular mappings in space $\mathbf{R}^{n}$ with dimension $n \geq 3$. It turns out this problem depends on the power $p$ of the underlying Sobolev space. For $p$ not too far below the dimension $n$ we show that a weakly quasiregular map in $W^{1, p}\left(\Omega ; \mathbf{R}^{n}\right)$ can only assume a quasiregular linear boundary value; however, for all $L \geq 1$ and $1 \leq p<\frac{n L}{L+1}$, we prove a rather surprising existence result that every linear map can be the boundary value of a weakly $L$-quasiregular map in $W^{1, p}\left(\Omega ; \mathbf{R}^{n}\right)$.
\end{abstract}

Mathematics Subject Classification (1991):30C65, 30C70, 35F30, 49J30

\section{Introduction}

In this paper, we use some techniques recently discovered in study of vectorial Hamilton-Jacobi equations in the calculus of variations to investigate the problem concerning the linear boundary values of weakly quasiregular mappings on a Lipschitz domain $\Omega$ in space $\mathbf{R}^{n}$ with dimension $n \geq 3$. The main results of the paper have been recently announced in Yan [28].

We recall that (see, e.g., Astala [2], Iwaniec [11], Iwaniec and Martin [14]) a map $u$ from a domain $\Omega$ in $\mathbf{R}^{n}$ to $\mathbf{R}^{n}$ is said to be weakly $L$ quasiregular, $L \geq 1$ being a constant called the (outer) dilatation of $u$, if it belongs to a (local) Sobolev space $W_{l o c}^{1, p}\left(\Omega ; \mathbf{R}^{n}\right)$ for some $p \geq 1$ and satisfies 


$$
|D u(x)|^{n} \leq L \operatorname{det} D u(x)
$$

for almost every $x \in \Omega$, where $D u=\left(\partial u^{i} / \partial x_{j}\right)$ denotes the gradient matrix of $u$ and $|\xi|$ denotes the matrix norm defined by $|\xi|=\max _{|h|=1}|\xi h|$. Note that the class of weakly quasiregular mappings in the standard space $W_{l o c}^{1, n}\left(\Omega ; \mathbf{R}^{n}\right)$ becomes the class of usual quasiregular mappings (see e.g. Rickman [24]) and also coincides with the class of mappings with bounded distortion (see Reshetnyak [23]). Weakly 1-quasiregular maps will be called the weakly conformal maps. Clearly, a weakly conformal map is weakly $L$ quasiregular for all $L>1$.

In order to study the weakly $L$-quasiregular mappings in the framework of vectorial Hamilton-Jacobi equations in the calculus of variations, we consider the $L$-quasiregular sets defined by (for any $L \geq 1$ )

$$
K_{L}=\left\{\left.\xi \in \mathbf{M}^{n \times n}|| \xi\right|^{n} \leq L \operatorname{det} \xi\right\},
$$

where $\mathbf{M}^{n \times n}$ denotes the real $n \times n$ matrices with norm $|\xi|$ defined above. When $L=1$, the set $K_{1}$ will be called the conformal set. A map $u \in$ $W_{l o c}^{1, p}\left(\Omega ; \mathbf{R}^{n}\right)$ is then weakly $L$-quasiregular if and only if it satisfies the special Hamilton-Jacobi equation

$$
D u(x) \in K_{L} \quad \text { a.e. } x \in \Omega .
$$

Closely related to the set $K_{L}$ is an important function $F_{L}$ on $\mathbf{M}^{n \times n}$ defined by

$$
F_{L}(\xi)=\max \left\{0,|\xi|^{n}-L \operatorname{det} \xi\right\} .
$$

On one hand, this function characterizes completely the class of weakly $L$-quasiregular mappings as mappings in $W_{l o c}^{1, p}\left(\Omega ; \mathbf{R}^{n}\right)$ that satisfy

$$
F_{L}(D u(x))=0 \quad \text { a.e. } x \in \Omega ;
$$

on the other hand, function $F_{L}$ satisfies an important condition of quasiconvexity introduced in Morrey [16] in the calculus of variations (see also Ball [3], Ball and Murat [5], Dacorogna [7], Morrey [17]); namely, $F_{L}$ satisfies

$$
\begin{gathered}
F_{L}(\xi) \leq \frac{1}{|\Omega|} \int_{\Omega} F_{L}(\xi+D \phi(x)) d x \\
\forall \xi \in \mathbf{M}^{n \times n}, \phi \in C_{0}^{\infty}\left(\Omega ; \mathbf{R}^{n}\right) .
\end{gathered}
$$

As an easy consequence of property (1.4), one easily shows that if a weakly $L$-quasiregular map $u$ in $W^{1, n}\left(\Omega ; \mathbf{R}^{n}\right)$ assumes an affine boundary value $\left.u\right|_{\partial \Omega}=\xi x+b$ then one must have $\xi \in K_{L}$.

One of the main results of this paper is to show this result is also valid for weakly $L$-quasiregular mappings in $W^{1, p}\left(\Omega ; \mathbf{R}^{n}\right)$ if $p$ is not too far below the dimension $n$. We prove the following theorem. 
Theorem 1.1. For each $L \geq 1$ there exists an index $p=p_{n}(L)<n$ such that if a weakly L-quasiregular map $u$ in $W^{1, p}\left(\Omega ; \mathbf{R}^{n}\right)$ assumes an affine boundary value $\left.u\right|_{\partial \Omega}=\xi x+b$ then $\xi \in K_{L}$.

Note that by our later existence theorem (see Theorem 1.3) and a recent significant conjecture of Iwaniec [12] the optimal number $p_{n}(L)$ would be given by $p_{n}(L)=\frac{n L}{L+1}$ for all $n \geq 3$ and $L \geq 1$. This can be shown to be the case if $L=1$ and $n$ is even; we have the following sharper result.

Theorem 1.2. Let dimension $n \geq 4$ be even. Suppose a weakly conformal map $u$ in $W^{1, n / 2}\left(\Omega ; \mathbf{R}^{n}\right)$ assumes an affine boundary value $\left.u\right|_{\partial \Omega}=\xi x+b$. Then $\xi \in K_{1}$ and $u \equiv \xi x+b$ a.e. in $\Omega$.

The proof of Theorem 1.1 and 1.2 relies on the existence of certain quasiconvex functions vanishing exactly on the set $K_{L}$. The existence of such quasiconvex functions has been established in Müller, Šverák and Yan [20], and Yan and Zhou [29], following important work of Iwaniec [11], Iwaniec and Sbordone [15] and Iwaniec and Martin [14].

The situation of linear boundary value problem may be completely different for weakly quasiregular mappings in Sobolev spaces $W^{1, p}\left(\Omega ; \mathbf{R}^{n}\right)$ for power $p$ not too close to the dimension $n$. We prove the following main existence result.

Theorem 1.3. Let $n \geq 3, L \geq 1$ and $1 \leq p<\frac{n L}{L+1}$. Then given any affine map $\xi x+b$ there exists a weakly L-quasiregular map $u$ in $W^{1, p}\left(\Omega ; \mathbf{R}^{n}\right)$ such that $\left.u\right|_{\partial \Omega}=\xi x+b$.

This result includes a completely new result even for weakly conformal mappings in $\mathbf{R}^{n}$ for all dimensions $n \geq 3$. We have the following special case of Theorem 1.3, which implies that for even dimensions $n$ the power $n / 2$ is optimal for the conclusion of Theorem 1.2.

Corollary 1.4. Let $n \geq 3$. Then every affine map can be the boundary value of a weakly conformal map in $W^{1, p}\left(\Omega ; \mathbf{R}^{n}\right)$ for any $1 \leq p<n / 2$.

More general boundary data can be considered for weakly quasiregular mappings. Throughout this paper, we say a map $\varphi \in W^{1, p}\left(\Omega ; \mathbf{R}^{n}\right)$ is piecewise affine if there exist at most countably many disjoint open subsets $\Omega_{j}$ of $\Omega$ whose union has full measure such that each $\left.\varphi\right|_{\Omega_{j}}$ is affine. We shall prove the following stronger version of Theorem 1.3.

Theorem 1.5. Let $n \geq 3, L \geq 1$ and $1 \leq p<\frac{n L}{L+1}$. Then, for any piecewise affine map $\varphi \in W^{1, p}\left(\Omega ; \mathbf{R}^{n}\right)$ and $\epsilon>0$, there exists a weakly $L$ quasiregular map $u_{\epsilon} \in \varphi+W_{0}^{1, p}\left(\Omega ; \mathbf{R}^{n}\right)$ such that $\left\|u_{\epsilon}-\varphi\right\|_{L^{p}(\Omega)}<\epsilon$. 
The proof of Theorem 1.3 relies on some important ideas derived from new investigations of Gromov's method of convex integration (see Gromov [10]). Such investigations have been initiated and successfully applied to the existence study of vectorial Hamilton-Jacobi equations of the form $D u(x) \in$ $K$ by Müller and Šverák [18], [19]; a similar method has been recently applied to the vectorial Hamilton-Jacobi equations of more general form $L(x, u(x), D u(x))=0$ by Müller and Sychev [21]. A different approach to the existence study for vectorial Hamilton-Jacobi equations uses the Baire category method and has been pursued by Dacorogna and Marcellini [8], [9].

In this paper, we establish a general existence theorem (Theorem 3.2) on the Hamilton-Jacobi equation $D u(x) \in K$ with affine boundary conditions for a given set $K$ which may be unbounded, a case not covered by the general study in papers [8], [9], [18], [19], [21] mentioned above. Our existence theorem is given in a form that is sufficient for the proof of Theorems 1.3 and 1.5; some generalization can be established to cover more general Hamilton-Jacobi equations but will not be included in the present paper.

\section{Notation and Preliminaries}

Before we proceed to prove the main theorems in following sections, we explain some notation and preliminaries.

Let $m, n \geq 1$ be integers. We use $\mathbf{M}^{m \times n}$ to denote the space of all real $m \times n$ matrices with the operator norm $|\xi|=\max _{|h|=1}|\xi h|$. We use rank $\xi$ to denote the rank of matrix $\xi$.

Given $a \in \mathbf{R}^{m}, b \in \mathbf{R}^{n}$, let $a \otimes b$ be the rank-one matrix with elements $(a \otimes b)_{i j}=a^{i} b^{j}$. If $m=n$, we use $\operatorname{det} \xi$ to denote the determinant of square matrix $\xi$ and also use $D=\operatorname{diag}\left(d_{1}, \cdots, d_{n}\right)$ to denote the diagonal matrix $D \in \mathbf{M}^{n \times n}$ with $d_{i i}=d_{i}$ and $d_{i j}=0$ for $i \neq j$.

We shall always assume $\Omega$ is a bounded open domain in $\mathbf{R}^{n}$ with Lipschitz boundary $\partial \Omega$. For any measurable set $E$ in $\mathbf{R}^{n}$ we use $|E|$ to denote its Lebesgue measure. We also use $\bar{E}, \operatorname{int} E, \operatorname{conv} E$ and $\chi_{E}$ to denote, respectively, closure, interior, closed convex hull and characteristic function of any given set $E$.

For $1 \leq p \leq \infty$, let $W^{1, p}\left(\Omega ; \mathbf{R}^{m}\right)$ be the usual Sobolev space of mappings from $\Omega$ to $\mathbf{R}^{m}$ with norm (see [1], [17])

$$
\|u\|_{W^{1, p}}=\|u\|_{L^{p}}+\|D u\|_{L^{p}}
$$

where $D u=\left(\partial u^{i} / \partial x_{j}\right), i=1, \cdots, m, j=1, \cdots, n$, is the gradient matrix of $u$. Let $W_{l o c}^{1, p}\left(\Omega ; \mathbf{R}^{m}\right)$ be the local Sobolev space. Note that for any Lipschitz domain $\Omega$ one can identify the space $W^{1, \infty}\left(\Omega ; \mathbf{R}^{m}\right)$ with the space of all Lipschitz continuous mappings from $\Omega$ to $\mathbf{R}^{m}$. We also denote 
by $W_{0}^{1, p}\left(\Omega ; \mathbf{R}^{m}\right)$ the completion of $C_{0}^{\infty}\left(\Omega ; \mathbf{R}^{m}\right)$, the set of all smooth maps with compact support in $\Omega$, in $W^{1, p}\left(\Omega ; \mathbf{R}^{m}\right)$ under the norm defined above.

Two maps $u, v$ in $W^{1, p}\left(\Omega ; \mathbf{R}^{m}\right)$ are said to have the same boundary value and write $\left.u\right|_{\partial \Omega}=\left.v\right|_{\partial \Omega}$ or $u \in v+W_{0}^{1, p}\left(\Omega ; \mathbf{R}^{m}\right)$ provided that $u-v \in W_{0}^{1, p}\left(\Omega ; \mathbf{R}^{m}\right)$.

Finally, we state a simple result frequently used throughout the paper; the proof is elementary and is left to the interested reader.

Lemma 1.6. Let $\left\{\Omega_{j}\right\}$ be a set of at most countably many disjoint open subsets of $\Omega$. Let $u \in W^{1, p}\left(\Omega ; \mathbf{R}^{m}\right)$ and $u_{j} \in W^{1, p}\left(\Omega_{j} ; \mathbf{R}^{m}\right)$ satisfy that $\left.u_{j}\right|_{\partial \Omega_{j}}=\left.u\right|_{\partial \Omega_{j}}$ for each index $j$. Suppose $\sum_{j}\left\|u_{j}\right\|_{W^{1, p}}^{p}<\infty$ if $p<\infty$ or $\sup _{j}\left\|u_{j}\right\|_{W^{1, \infty}}<\infty$ if $p=\infty$. Then the map $\tilde{u}=\chi_{\Omega \backslash \cup_{j} \Omega_{j}} u+\sum_{j} \chi_{\Omega_{j}} u_{j}$ belongs to $u+W_{0}^{1, p}\left(\Omega ; \mathbf{R}^{m}\right)$.

\section{Quasiconvex functions vanishing on quasiregular sets}

In this section, we aim to prove Theorems 1.1 and 1.2.

Let $F: \mathbf{M}^{m \times n} \rightarrow \mathbf{R}$ be a function. According to Morrey [16], $F$ is said to be quasiconvex on $\mathbf{M}^{m \times n}$ provided the property

$$
F(\xi) \leq \frac{1}{|D|} \int_{D} F(\xi+D \varphi(x)) d x
$$

holds for all $\xi \in \mathbf{M}^{m \times n}$, bounded domains $D \subset \mathbf{R}^{n}$, and all smooth maps $\varphi \in C_{0}^{\infty}\left(D ; \mathbf{R}^{m}\right)$. Given any function $f: \mathbf{M}^{m \times n} \rightarrow \mathbf{R}$, we define the quasiconvexification of $f$, denoted by $f^{q c}$, to be the function given by

$$
f^{q c}(\xi)=\inf _{\varphi \in C_{0}^{\infty}\left(D ; \mathbf{R}^{m}\right)} \frac{1}{|D|} \int_{D} f(\xi+D \varphi(x)) d x .
$$

It is well-known that the function $f^{q c}$ is independent of the domain $D$ and is always quasiconvex assuming $f$ is continuous; see, e.g., [3], [7], [16], [17].

If $F: \mathbf{M}^{m \times n} \rightarrow \mathbf{R}$ is quasiconvex function and satisfies

$$
|F(\xi)| \leq C\left(|\xi|^{p}+1\right) \quad \xi \in \mathbf{M}^{m \times n},
$$

where $C>0, p \geq 1$ are some constants, we observe that, by a density argument (see e.g. [5], [7], [17]), property (2.1) also holds for all $\varphi \in$ $W_{0}^{1, p}\left(D ; \mathbf{R}^{m}\right)$. From this observation, we easily have the following result.

Proposition 2.1. Let $F: \mathbf{M}^{m \times n} \rightarrow \mathbf{R}$ be quasiconvex and satisfy (2.3). Suppose $K \subset \mathbf{M}^{m \times n}$ is a set such that $\left.F\right|_{K}=0$. If $u \in W^{1, p}\left(\Omega ; \mathbf{R}^{m}\right)$ satisfies $\left.u\right|_{\partial \Omega}=\xi x+b$ and $F(D u(x))=0$ a.e. in $\Omega$, then one has $F(\xi) \leq 0$. 
We now consider the $L$-quasiregular set $K_{L} \subset \mathbf{M}^{n \times n}$ defined above by (1.2). As defined by (1.3), let $F_{L}: \mathbf{M}^{n \times n} \rightarrow \mathbf{R}$ be the function given by

$$
F_{L}(\xi)=\max \left\{0,|\xi|^{n}-L \operatorname{det} \xi\right\} .
$$

Then, by (1.4), $F_{L}$ is quasiconvex and one easily sees that $F_{L}$ vanishes exactly on $K_{L}$; note also that the growth of $F_{L}$ is of $n$-th power: $0 \leq$ $F_{L}(\xi) \leq C|\xi|^{n}$.

Another important property of this function $F_{L}$ is that it easily satisfies the so-called $L^{n}$-mean coercivity condition (see, e.g., [13], [30]):

$$
\int_{D} F_{L}(D \varphi(x)) d x \geq \int_{D}|D \varphi(x)|^{n} d x \quad \forall \varphi \in C_{0}^{\infty}\left(D ; \mathbf{R}^{m}\right) .
$$

Existence of the quasiconvex functions with growth below the natural power $n$ and vanishing exactly on the quasiregular set is the main content of our next theorem; we refer to [20], [30] for the proof and further discussions.

Theorem 2.2 ([20], [30]). Let $n \geq 3$. Then for every $L \geq 1$ there exists a power $p_{n}(L) \in[n / 2, n)$ such that for each $p \geq p_{n}(L)$ one can always have a quasiconvex function $g$ satisfying

$$
0 \leq g(\xi) \leq|\xi|^{p}, \quad g(\xi)=0 \Longleftrightarrow \xi \in K_{L} .
$$

Furthermore, if $n$ is even and $L=1$, the optimal power $p_{n}(1)$ equals $n / 2$.

Remark. 1) Existence of quasiconvex functions satisfying (2.5) follows from property (2.4) and general results established in Yan and Zhou [30] using the important technique of nonlinear Hodge decomposition discovered in Iwaniec [11] and Iwaniec and Sbordone [15]. In fact, by [30], Theorem 2.1 , one can choose $g$ to be the quasiconvexification function $\left(F_{L}^{p / n}\right)^{q c}$ for $p \geq p_{n}(L)$.

2) The special case when $n$ is even and $L=1$ has been considered by Müller, Šverák and Yan in [20], using a special linear structure of the conformal set $K_{1}$ and following the important work of Iwaniec and Martin [14].

3) In view of some recent results in Iwaniec [12] (see also Astala [2]), we conjecture that the optimal power $p_{n}(L)$ in the theorem is given by $p_{n}(L)=\frac{n L}{L+1}$ for all $n \geq 3, L \geq 1$.

\section{Proof of Theorem 1.1}

Let $p_{n}(L)<n$ be the number determined in Theorem 2.2 and let $g$ be the function satisfying (2.5) with $p=p_{n}(L)$. Assume $u$ is a weakly $L$ quasiregular map in $\xi x+b+W_{0}^{1, p}\left(\Omega ; \mathbf{R}^{n}\right)$, where $p=p_{n}(L)$. Since $g(D u(x))=0$ a.e. in $\Omega$, by Proposition 2.1, one has $g(\xi) \leq 0$ and hence $\xi \in K_{L}$, as claimed. This completes the proof. 


\section{Proof of Theorem 1.2}

Let $n \geq 4$ be even, and let $u \in W^{1, n / 2}\left(\Omega ; \mathbf{R}^{n}\right)$ be a weakly conformal map and $\left.u\right|_{\partial \Omega}=\xi x+b$. As in the proof of Theorem 1.1 it follows from Proposition 2.1 and Theorem 2.2 that $\xi \in K_{1}$. Therefore the map $\tilde{u}=\chi_{\Omega} u+$ $\chi_{\mathbf{R}^{n} \backslash \Omega}(\xi x+b)$ is a weakly conformal map in $W_{l o c}^{1, n / 2}\left(\mathbf{R}^{n} ; \mathbf{R}^{n}\right)$. By a wellknown result of Iwaniec and Martin [14], $\tilde{u}$ must belong to $W_{l o c}^{1, n}\left(\mathbf{R}^{n} ; \mathbf{R}^{n}\right)$. Then a classical result of Liouville's theorem (see Reshetnyak [23]) shows $\tilde{u}$ is a Möbius transformation; hence, $\tilde{u} \equiv \xi x+b$. The proof of Theorem 1.2 is now completed.

\section{A general existence theorem}

In this section we establish an existence theorem for vectorial HamiltonJacobi equation of the form

$$
D u(x) \in K, \quad \text { a.e. } x \in \Omega,
$$

where $K$ is a given subset of $\mathbf{M}^{m \times n}$. In studying equations of this type, it is important to investigate certain special structures of the set $K$.

Definition 3.1. Given any set $K \subset \mathbf{M}^{m \times n}$, define sets $\mathcal{L}_{j}(K)$ for $j=$ $0,1,2, \cdots$ inductively as follows: $\mathcal{L}_{0}(K)=K$ and, for $j=0,1, \cdots$, $\mathcal{L}_{j+1}(K)=\left\{t \xi+(1-t) \eta \mid t \in[0,1], \xi, \eta \in \mathcal{L}_{j}(K), \operatorname{rank}(\xi-\eta) \leq 1\right\}$. Define the lamination hull of set $K$ to be the set given by

$$
\mathcal{L}(K)=\cup_{j=0}^{\infty} \mathcal{L}_{j}(K) .
$$

Remark. Clearly, from the definition, $\mathcal{L}_{j+1}(K)=\mathcal{L}_{1}\left(\mathcal{L}_{j}(K)\right) \supseteq \mathcal{L}_{j}(K)$ for all $j=0,1, \cdots$. We refer to, e.g., [4], [7], [8], [9], [18], [19], [22], [29], [30], [31] for more properties of this and other generalized convex hulls in the calculus of variations.

Definition 3.2. For each $1 \leq p \leq \infty$, define $\beta_{p}(K)$ to be the set of all matrices $\xi$ in $\mathbf{M}^{m \times n}$ such that there exists a map $u=u_{\xi} \in W^{1, p}\left(\Omega ; \mathbf{R}^{m}\right)$ satisfying

$$
D u(x) \in K \quad \text { a.e. } x \in \Omega,\left.\quad u\right|_{\partial \Omega}=\xi x .
$$

Remark. 1) If $\xi \in K$, we can always choose $u_{\xi} \equiv \xi x$; hence $K \subseteq \beta_{p}(K)$. Clearly, we always have $\beta_{p}(K) \subseteq \beta_{q}(K)$ for any $1 \leq q<p \leq \infty$.

2) The following result shows that the set $\beta_{p}(K)$ is independent of the domain $\Omega$ and that the map $u \in \xi x+W_{0}^{1, p}\left(\Omega ; \mathbf{R}^{m}\right)$ can be chosen in such a way that $\|u-\xi x\|_{L^{p}(\Omega)}$ is arbitrarily small. 
Lemma 3.1. Let $\xi \in \beta_{p}(K)$ and let $u_{\xi}$ be the map in the definition above. Then, for any bounded open set $\Sigma$ in $\mathbf{R}^{n}$ and any $\epsilon>0$, there exists a map $v(y) \in \xi y+W_{0}^{1, p}\left(\Sigma ; \mathbf{R}^{m}\right)$ such that $\|v-\xi y\|_{L^{p}(\Sigma)}<\epsilon$ and

$$
\frac{1}{|\Sigma|} \int_{\Sigma} g(D v(y)) d y=\frac{1}{|\Omega|} \int_{\Omega} g\left(D u_{\xi}(x)\right) d x
$$

for any continuous function $g$ with $|g(\eta)| \leq C\left(|\eta|^{p}+1\right)$.

Proof. Without loss of generality, assume $0 \in \Omega$. Fix a number $\delta>0$. Since $\Sigma$ is open, for any $y \in \Sigma$, there exists $\epsilon_{y}$ between 0 and $\delta$ such that the sets $\Omega_{y, \epsilon} \equiv y+\epsilon \bar{\Omega}$ are contained in $\Sigma$ for all $0<\epsilon<\epsilon_{y}$. Note that all such sets $\left\{\Omega_{y, \epsilon}\right\}$ form a Vitali cover of $\Sigma$; hence, by the Vitali covering lemma, there exist disjoint sets $\left\{y_{k}+\epsilon_{k} \bar{\Omega}\right\}$ and a null set $N$ such that $0<\epsilon_{k}<\delta$ and $\Sigma=\cup_{k}\left(y_{k}+\epsilon_{k} \bar{\Omega}\right) \cup N$. Define $v=v^{\delta}: \Sigma \rightarrow \mathbf{R}^{n}$ by

$$
v^{\delta}(y)= \begin{cases}\xi y+\epsilon_{k} u_{\xi}\left(\frac{y-y_{k}}{\epsilon_{k}}\right) & \text { if } y \in y_{k}+\epsilon_{k} \Omega \text { for some } k \\ \xi y & \text { otherwise in } \Sigma .\end{cases}
$$

Then it is easy to see that $v^{\delta}(y) \in \xi y+W_{0}^{1, p}\left(\Sigma ; \mathbf{R}^{m}\right)$. Easy computation also shows that

$$
\frac{1}{|\Sigma|} \int_{\Sigma} g\left(D v^{\delta}(y)\right) d y=\frac{1}{|\Omega|} \int_{\Omega} g\left(D u_{\xi}(x)\right) d x
$$

for any continuous function $g$ with $|g(\eta)| \leq C\left(|\eta|^{p}+1\right)$. Finally, by choosing $\delta>0$ sufficiently small, one can make $\left\|v^{\delta}-\xi y\right\|_{L^{p}(\Sigma)}<\epsilon$.

We now state our main existence theorem of this section.

Theorem 3.2. Let $K \subset \mathbf{M}^{m \times n}$ be a closed set and let $A \subset \beta_{p}(K)$ be a set satisfying

$$
c_{0}=\sup _{\xi \in A} \frac{1}{|\Omega|} \int_{\Omega}\left|D u_{\xi}\right|^{p} d x<\infty,
$$

where $u_{\xi} \in W^{1, p}\left(\Omega ; \mathbf{R}^{m}\right)$ is some map satisfying (3.2). Suppose the lamination hull $B=\mathcal{L}(A)$ is open and bounded. Then $B \subset \beta_{p}(K)$.

From this theorem, we easily have the following result; we refer to Müller and Šverák [18] for similar results about open relations and to Gromov [10] and Müller and Sychev[21] for other related results.

Corollary 3.3. Let $A \subset \mathbf{M}^{m \times n}$ be a bounded set such that $\mathcal{L}(A)$ is open. Then $\mathcal{L}(A) \subset \beta_{\infty}(\bar{A})$.

Proof. The result follows easily from the theorem with $K=\bar{A}$. 
The following result is essential for proving our general existence theorem. Other versions of the result can be found in [8], [9], [18], [19] and [21]; a similar result in the case $m=1$ has been used by Cellina in [6].

Lemma 3.4. Let $U$ be an open set in $\mathbf{M}^{m \times n}$ and let $\eta \in U$ and $\eta=$ $t \eta_{1}+(1-t) \eta_{2}$ with $t \in(0,1)$ and $\operatorname{rank}\left(\eta_{1}-\eta_{2}\right)=1$. Then, for any $\epsilon>0$, there exist piece-wise affine map $u \in \eta x+W_{0}^{1, \infty}\left(\Omega ; \mathbf{R}^{m}\right)$ and finitely many points $\eta_{3}, \cdots, \eta_{s}$ in $U$ such that $D u(x) \in\left\{\eta_{1}, \eta_{2}, \eta_{3}, \cdots, \eta_{s}\right\}$ a.e. in $\Omega$ and the measure of the set $\left\{x \in \Omega \mid D u(x) \neq \eta_{1}, D u(x) \neq \eta_{2}\right\}$ is less than $\epsilon$.

Proof. The proof follows closely some ideas in [6], [21]. We proceed in several steps.

1. Let $\eta_{1}=\eta_{2}+a \otimes b$, where $a \in \mathbf{R}^{m}, b \in \mathbf{R}^{n}$ and $|b|=1$. Then $\eta_{1}-\eta=a \otimes b_{1}, \eta_{2}-\eta=a \otimes b_{2}$, where $b_{1}=(1-t) b, b_{2}=-t b$. Since $\eta \in U$ and $U$ is open, we can choose $b_{3}, \cdots, b_{s}$ in $\mathbf{R}^{n}$ such that $\eta_{j} \equiv \eta+a \otimes b_{j} \in U$ for all $j=3, \cdots, s$, each $b_{j}$ is extreme point of the convex hull $H \equiv \operatorname{conv}\left\{b_{1}, b_{2}, \cdots, b_{s}\right\}$, and $0 \in \operatorname{int} H$. Note that we can choose $s=n+2$.

2. For each $k=1,2, \cdots$, let $\left\{b_{3}^{k}, \cdots, b_{s}^{k}\right\}$ be a set of points contained in int $H$ such that $\left|b_{j}^{k}-b_{1}\right|<1 / k$ and $0 \in \operatorname{intconv}\left\{b_{1}, b_{2}, b_{3}^{k}, \cdots, b_{s}^{k}\right\}$ for all $k$. For simplicity, we also write $b_{i}=b_{i}^{k}$ for $i=1,2$ and $k=1,2, \cdots$.

3 . For each given $k=1,2, \cdots$, consider the function

$$
w_{k}(x)=-1+\max _{1 \leq j \leq s} b_{j}^{k} \cdot x, \quad x \in \mathbf{R}^{n} .
$$

It is clear that $w_{k}$ is piece-wise affine, Lipschitz continuous on $\mathbf{R}^{n}$ and satisfies $D w_{k} \in\left\{b_{1}, b_{2}, b_{3}^{k}, \cdots, b_{s}^{k}\right\}$ a.e. in $\mathbf{R}^{n}$. From the fact that $0 \in$ intconv $\left\{b_{1}^{k}, \cdots, b_{s}^{k}\right\}$, the set $\Sigma=\left\{x \in \mathbf{R}^{n} \mid w_{k}(x)<0\right\}$ is a bounded open polyhedral convex set containing 0 and $\left.w_{k}\right|_{\partial \Sigma}=0$ (see e.g. [25]). Therefore, we have proved that there exist a Lipschitz bounded domain $\Sigma$ in $\mathbf{R}^{n}$ and a piece-wise affine function $w=w_{k} \in W_{0}^{1, \infty}(\Sigma)$ such that $D w \in\left\{b_{1}, b_{2}, b_{3}^{k}, \cdots, b_{s}^{k}\right\}$ a.e. in $\Sigma$.

4. A similar argument using the Vitali covering lemma as the one used in the proof of Lemma 3.1 shows that there exists a piece-wise affine function $h_{k} \in W_{0}^{1, \infty}(\Omega)$ with $D h_{k} \in\left\{b_{1}, b_{2}, b_{3}^{k}, \cdots, b_{s}^{k}\right\}$ a.e. in $\Omega$. Note that it is important here that we allow our piece-wise affine maps to be affine on countably many sets. For each $j=1,2, \cdots, s$, define $\Omega_{j}^{k}=\{x \in$ $\left.\Omega \mid D h_{k}(x)=b_{j}^{k}\right\}$.

5. We modify the values of $h_{k}$ on the set $\Omega_{j}^{k}$ for each $j=3, \cdots, s$ so that the gradients belong to the set $\left\{b_{1}, b_{2}, \cdots, b_{s}\right\}$. To do this, let $j \in$ $\{3, \cdots, s\}$ be given, and let $\Omega_{j}^{k}=\sum_{\nu=1}^{\infty} \Sigma_{j \nu}^{k}$ be such that $\left.h_{k}\right|_{\sum_{j \nu}^{k}}$ is affine and $\left.D h_{k}\right|_{\Sigma_{j \nu}^{k}}=b_{j}^{k}$ for all $\nu=1, \cdots$. Since $b_{j}^{k} \in \operatorname{intconv}\left\{b_{1}, b_{2}, \cdots, b_{s}\right\}$, we have $0 \in \operatorname{intconv}\left\{b_{1}-b_{j}^{k}, \cdots, b_{s}-b_{j}^{k}\right\}$. As in Steps 3 and 4 , we have a 
piece-wise affine Lipschitz function $\tilde{h}_{\nu} \in W_{0}^{1, \infty}\left(\Sigma_{j \nu}^{k}\right)$ such that $D \tilde{h}_{\nu}(x) \in$ $\left\{b_{1}-b_{j}^{k}, b_{2}-b_{j}^{k}, \cdots, b_{s}-b_{j}^{k}\right\}$ a.e. in $\Sigma_{j \nu}^{k}$. Define $v_{j}^{k}=h_{k}+\sum_{\nu=1}^{\infty} \chi_{\Sigma_{j \nu}^{k}} \tilde{h}_{\nu}$ on $\Omega_{j}^{k}$. Then $v_{j}^{k} \in h_{k}+W_{0}^{1, \infty}\left(\Omega_{j}^{k}\right)$ and $D v_{j}^{k} \in\left\{b_{1}, b_{2}, \cdots, b_{s}\right\}$ a.e. in $\Omega_{j}^{k}$. Let

$$
G_{i j}^{k}=\left\{x \in \Omega_{j}^{k} \mid D v_{j}^{k}(x)=b_{i}\right\}, \quad \sigma_{i j}^{k}=\left|G_{i j}^{k}\right| /\left|\Omega_{j}^{k}\right| .
$$

Since $v_{j}^{k} \in h_{k}+W_{0}^{1, \infty}\left(\Omega_{j}^{k}\right)$, we easily see that for all $k=1,2, \cdots$

$$
b_{j}^{k}=\sum_{i=1}^{s} \sigma_{i j}^{k} b_{i}, \quad \sum_{i=1}^{s} \sigma_{i j}^{k}=1 .
$$

Note that, for any $j \geq 3, b_{j}^{k} \rightarrow b_{1}$ as $k \rightarrow \infty$ and, by the assumption of our selection, $b_{1}$ is an extreme point of $\operatorname{conv}\left\{b_{1}, b_{2}, \cdots, b_{s}\right\}$. We easily obtain from (3.5) that for each $j=3, \cdots, s$

$$
\lim _{k \rightarrow \infty} \sigma_{i j}^{k}=0, \quad \forall i=2,3, \cdots, s .
$$

6. Define $f_{k}=\chi_{\Omega_{1}^{k} \cup \Omega_{2}^{k}} h_{k}+\sum_{j=3}^{s} \chi_{\Omega_{j}^{k}} v_{j}^{k}$ on $\Omega$. Then, $f_{k}$ is piece-wise affine and belongs to $W_{0}^{1, \infty}(\Omega)$, and one has $D f_{k}(x) \in\left\{b_{1}, b_{2}, \cdots, b_{s}\right\}$ a.e. in $\Omega$. Note that, for any $i=3, \cdots, s$, the set $\left\{x \in \Omega \mid D f_{k}(x)=b_{i}\right\}$ equals (up to a set of measure zero) $\cup_{j=3}^{s} G_{i j}^{k}$, where sets $G_{i j}^{k}$ are defined in Step 5 . Hence, by (3.6), we have

$$
\lim _{k \rightarrow \infty}\left|\left\{x \in \Omega \mid D f_{k}(x)=b_{i}\right\}\right|=0 \quad \forall i \geq 3 .
$$

This proves the measure of the set $\left\{x \in \Omega \mid D f_{k}(x) \notin\left\{b_{1}, b_{2}\right\}\right\}$ tends to zero as $k \rightarrow \infty$. We choose a large $\bar{k}$ such that $\mid\left\{x \in \Omega \mid D f_{\bar{k}}(x) \notin\right.$ $\left.\left\{b_{1}, b_{2}\right\}\right\} \mid<\epsilon$.

7. Finally, let $u(x) \equiv \eta x+f_{\bar{k}}(x) a$ for $x \in \Omega$. We easily see that $u$ is a piece-wise affine map and satisfies all the requirements of the lemma. This completes the proof.

Proposition 3.5. Let $B=\mathcal{L}(A)$ be an open set in $\mathbf{M}^{m \times n}$. Then for any $\xi \in$ $B$ and $\epsilon>0$, there exist a piece-wise affine map $u \in \xi x+W_{0}^{1, \infty}\left(\Omega ; \mathbf{R}^{m}\right)$ and two sets of finitely many points $\left\{\alpha_{1}, \alpha_{2}, \cdots, \alpha_{r}\right\} \subset A$ and $\left\{\xi_{1}, \xi_{2}, \cdots\right.$, $\left.\xi_{q}\right\} \subset B$ such that

$$
D u(x) \in\left\{\alpha_{1}, \cdots, \alpha_{r}\right\} \cup\left\{\xi_{1}, \cdots, \xi_{q}\right\}, \quad \text { a.e. } x \in \Omega
$$

and the measure $\left|\left\{x \in \Omega \mid D u(x) \notin\left\{\alpha_{1}, \alpha_{2}, \cdots, \alpha_{r}\right\}\right\}\right|<\epsilon$. 
Proof. Given $\xi \in B=\mathcal{L}(A)$, we have $\xi \in \mathcal{L}_{k}(A) \subset B$, where $k$ is the minimal such index. Then $\xi=t \eta_{1}+(1-t) \eta_{2}$ for some $t \in(0,1)$ and $\eta_{1}, \eta_{2} \in \mathcal{L}_{k-1}(A)$ with $\operatorname{rank}\left(\eta_{1}-\eta_{2}\right)=1$. Since $B$ is open and $\xi \in B$, by Lemma 3.1, we have a map $u_{1} \in \xi x+W_{0}^{1, \infty}\left(\Omega ; \mathbf{R}^{m}\right)$ and a set $\left\{\eta_{3}, \cdots, \eta_{s}\right\} \subset B$ such that

$$
\begin{gathered}
D u_{1}(x) \in\left\{\eta_{1}, \eta_{2}\right\} \cup\left\{\eta_{3}, \cdots, \eta_{s}\right\} \quad \text { a.e. } x \in \Omega ; \\
\left|\left\{x \in \Omega \mid D u_{1}(x) \notin\left\{\eta_{1}, \eta_{2}\right\}\right\}\right|<\epsilon_{1}<\epsilon .
\end{gathered}
$$

If $k=1$, the result is proved since then $\eta_{1}, \eta_{2} \in A$. If $k \geq 2$, we can apply similar arguments to each $\eta_{j} \in \mathcal{L}_{k-1}(A) \subset B$ and to each component of the set $\Omega_{j}=\left\{x \in \Omega \mid D u_{1}(x)=\eta_{j}\right\}$ where $u_{1}$ is affine to adjust the value $D u_{1}(x)=\eta_{j}$ to values in the set $\mathcal{L}_{k-2}(A)$ for most $x \in \Omega_{j}$, where $j=1,2$. Repeating this argument in a finite number of steps, one can eventually reach at the conclusion of the proposition.

Assume now $A \subset \beta_{p}(K)$ satisfies (3.3) and $B=\mathcal{L}(A)$ is open.

Let $\xi \in B$ and let $u$ be a map determined in the previous proposition. Let $\Sigma=\{x \in \Omega \mid D u(x) \in A\}=\cup_{j=1}^{\infty} \Sigma_{j}$, where $\left.u\right|_{\Sigma_{j}}=\alpha_{i(j)} x+d_{j}$ is affine for some $1 \leq i(j) \leq r$. By Lemma 3.1 and condition (3.3), there exists $v_{j} \in u+W_{0}^{1, p}\left(\Sigma_{j} ; \mathbf{R}^{m}\right)$ such that $D v_{j}(x) \in K$ a.e. in $\Sigma_{j}$ and $\int_{\Sigma_{j}}\left|D v_{j}\right|^{p} d x \leq c_{0}\left|\Sigma_{j}\right|$.

Let $\tilde{u}=\chi_{\Omega \backslash \Sigma} u+\sum_{j=1}^{\infty} \chi_{\Sigma_{j}} v_{j}$. Then $\tilde{u} \in \xi x+W_{0}^{1, p}\left(\Omega ; \mathbf{R}^{m}\right)$ and satisfies

$$
\begin{gathered}
D \tilde{u}(x) \in K \cup\left\{\xi_{1}, \cdots, \xi_{q}\right\} ; \\
\Omega_{\epsilon}=\left\{x \in \Omega \mid D \tilde{u}(x) \in\left\{\xi_{1}, \cdots, \xi_{q}\right\}\right\} ; \\
\left|\Omega_{\epsilon}\right|<\epsilon ; \\
\int_{\Omega \backslash \Omega_{\epsilon}}|D \tilde{u}|^{p} d x \leq c_{0}\left|\Omega \backslash \Omega_{\epsilon}\right| .
\end{gathered}
$$

\section{Proof of Theorem 3.2}

The proof is based on the previous construction and the boundedness assumption on $B=\mathcal{L}(A)$. We assume, for a constant $\lambda<\infty,|\eta|<\lambda$ for all $\eta \in B$.

Let $\xi \in B$ be given. We use the construction described above. Note that, in addition to (3.7)-(3.10), it also follows that $\int_{\Omega_{\epsilon}}|D \tilde{u}|^{p} d x \leq \lambda^{p}\left|\Omega_{\epsilon}\right|$.

In the following, let $\epsilon_{k} \rightarrow 0^{+}$be a decreasing sequence satisfying $\sum_{k} \epsilon_{k}^{1 / p}<\infty$.

Let $u_{1} \in \xi x+W_{0}^{1, p}\left(\Omega ; \mathbf{R}^{m}\right)$ be the function $\tilde{u}$ defined above with $\epsilon=\epsilon_{1}$. We modify the values of $u_{1}$ on each open set where $D u_{1} \notin K$. Let $\Omega_{1}=\Omega_{\epsilon}$ be the set defined in the construction. Write $\Omega_{1}=\cup_{j=1}^{\infty} \Delta_{j}$ 
such that $\left.u_{1}\right|_{\Delta_{j}}=\xi_{j} x+d_{j}$ and $\xi_{j} \in B$. For each $j$, we use the previous construction for $\xi_{j} \in B$ with domain $\Delta_{j}$ and number $\epsilon=\epsilon_{2} / 2^{j}$ to obtain a map $\tilde{v}_{j} \in u_{1}+W_{0}^{1, p}\left(\Delta_{j} ; \mathbf{R}^{m}\right)$ such that

$$
\begin{gathered}
D \tilde{v}_{j}(x) \in K \cup\left\{\xi_{1}^{\prime}, \cdots, \xi_{q^{\prime}}^{\prime}\right\} ; \\
\Delta_{j}^{\prime}=\left\{x \in \Delta_{j} \mid D \tilde{v}_{j}(x) \in\left\{\xi_{1}^{\prime}, \cdots, \xi_{q^{\prime}}^{\prime}\right\}\right\} ; \\
\left|\Delta_{j}^{\prime}\right|<\epsilon_{2} / 2^{j} ; \\
\int_{\Delta_{j} \backslash \Delta_{j}^{\prime}}\left|D \tilde{v}_{j}\right|^{p} d x \leq c_{0}\left|\Delta_{j} \backslash \Delta_{j}^{\prime}\right| .
\end{gathered}
$$

Let $\Omega_{2}=\cup_{j=1}^{\infty} \Delta_{j}^{\prime}$. Then $\left|\Omega_{2}\right|<\epsilon_{2}$. Define $u_{2}=\chi_{\Omega \backslash \Omega_{1}} u_{1}+\sum_{j=1}^{\infty} \chi_{\Delta_{j}} \tilde{v}_{j}$. Then $u_{2} \in \xi x+W_{0}^{1, p}\left(\Omega ; \mathbf{R}^{m}\right)$ satisfies that $u_{2}=u_{1}$ on $\Omega \backslash \Omega_{1}, D u_{2}(x) \in$ $B$ a.e. in $\Omega_{2}, D u_{2}(x) \in K$ for a.e. in $\Omega \backslash \Omega_{2}$, and $\int_{\Omega_{1} \backslash \Omega_{2}}\left|D u_{2}\right|^{p} d x \leq$ $c_{0}\left|\Omega_{1} \backslash \Omega_{2}\right|$.

We then modify the values of $u_{2}$ on the set $\Omega_{2}$ as we did for $u_{1}$ on $\Omega_{1}$ to obtain $u_{3}$ and $\Omega_{3}$. Continuing in this way, we obtain a sequence $\left\{u_{k}\right\}$ in $\xi x+W_{0}^{1, p}\left(\Omega ; \mathbf{R}^{m}\right)$ and open sets $\Omega_{k} \subset \Omega_{k-1} \subset \Omega$ such that

$$
\begin{gathered}
\left|\Omega_{k}\right|<\epsilon_{k} ; \\
D u_{k}(x) \in B \quad \text { a.e. in } \Omega_{k} ; \\
D u_{k}(x) \in K \quad \text { a.e. in } \Omega \backslash \Omega_{k} ; \\
u_{k+1}=u_{k} \quad \text { on } \Omega \backslash \Omega_{k} ; \\
\int_{\Omega_{k} \backslash \Omega_{k+1}}\left|D u_{k+1}\right|^{p} d x \leq c_{0}\left|\Omega_{k} \backslash \Omega_{k+1}\right| .
\end{gathered}
$$

First of all, note that conditions (3.16), (3.19) yield

$$
\int_{\Omega_{k}}\left|D u_{k+1}\right|^{p} d x \leq c_{0}\left|\Omega_{k} \backslash \Omega_{k+1}\right|+\lambda^{p}\left|\Omega_{k+1}\right| \leq C_{0}\left|\Omega_{k}\right|,
$$

where $C_{0}=\max \left\{c_{0}, \lambda^{p}\right\}$. Hence, by (3.18),

$$
\begin{aligned}
\left\|D u_{k+1}-D u_{k}\right\|_{L^{p}(\Omega)} & =\left\|D u_{k+1}-D u_{k}\right\|_{L^{p}\left(\Omega_{k}\right)} \\
& \leq 2 C_{0}^{1 / p}\left|\Omega_{k}\right|^{1 / p} .
\end{aligned}
$$

Furthermore, by (3.15)-(3.19), we easily obtain that

$$
\begin{gathered}
\int_{\Omega}\left|D u_{k+1}\right|^{p} d x \leq C_{0}|\Omega| ; \\
\int_{\Omega} \operatorname{dist}^{p}\left(D u_{k}(x) ; K\right) d x \leq C^{\prime}\left|\Omega_{k}\right|<C^{\prime} \epsilon_{k},
\end{gathered}
$$

where $C^{\prime}$ is a constant and $\operatorname{dist}(\eta ; K)$ is the distance function to the set $K$.

Finally, conditions (3.20), (3.21) and the convergence of $\sum_{k} \epsilon_{k}^{1 / p}$ imply that the sequence $\left\{u_{k}\right\}$ is a Cauchy sequence in $\xi x+W_{0}^{1, p}\left(\Omega ; \mathbf{R}^{m}\right)$. Let $\bar{u} \in \xi x+W_{0}^{1, p}\left(\Omega ; \mathbf{R}^{m}\right)$ be the limit of this sequence. Since $K$ is closed, 
condition (3.22) implies $D \bar{u}(x) \in K$ for almost every $x \in \Omega$. This proves $\xi \in \beta_{p}(K)$; hence, $B \subset \beta_{p}(K)$.

The proof of Theorem 3.2 is now completed.

Remark. From (3.21) in the proof above we easily see that the solution $\bar{u} \in \xi x+W_{0}^{1, p}\left(\Omega ; \mathbf{R}^{m}\right)$ obtained in the proof also satisfies

$$
\frac{1}{|\Omega|} \int_{\Omega}|D \bar{u}|^{p} d x \leq C_{0} \equiv \max \left\{c_{0}, \sup _{\eta \in B}|\eta|^{p}\right\} .
$$

\section{Linear boundary values of weakly quasiregular mappings}

In this section, we prove main results Theorems 1.3 and 1.5.

To apply the general existence theorem proved before, we introduce some notations.

Given a number $\lambda>0$, define

$$
\begin{gathered}
R(n)=\left\{\left.\xi \in \mathbf{M}^{n \times n}|| \xi\right|^{n}=|\operatorname{det} \xi|\right\} ; \\
A_{\lambda}=\{\xi \in R(n)|| \xi \mid<\lambda\} ; \\
B_{\lambda}=\left\{\xi \in \mathbf{M}^{n \times n}|| \xi \mid<\lambda\right\} ; \\
S O(n)=\{\xi \in R(n) \mid \operatorname{det} \xi=1\} .
\end{gathered}
$$

It is easy to see that the conformal set $K_{1}=\{\xi \in R(n) \mid \operatorname{det} \xi \geq 0\}$.

We have the following result.

Proposition 4.1. $\mathcal{L}_{n-1}\left(A_{\lambda}\right)=B_{\lambda}$. Therefore $B_{\lambda}=\mathcal{L}\left(A_{\lambda}\right)$ is an open set.

Proof. The proof follows from a refinement of the argument in Yan [27]. Since $A_{\lambda} \subset B_{\lambda}$ and $B_{\lambda}$ is convex and hence $\mathcal{L}\left(A_{\lambda}\right) \subseteq B_{\lambda}$, it suffices to show $B_{\lambda} \subseteq \mathcal{L}_{n-1}\left(A_{\lambda}\right)$. Let $\xi \in B_{\lambda}, \xi \neq 0$. By singular value decompositions of matrices, there exist $Q_{1}, Q_{2} \in S O(n)$ such that $\xi=$ $Q_{1} \operatorname{diag}\left(\sigma_{1}, \cdots, \sigma_{n}\right) Q_{2}$, where $\left|\sigma_{1}\right| \leq \cdots \leq\left|\sigma_{n}\right|, \sigma_{n} \neq 0$ and $\left|\sigma_{n}\right|<\lambda$. Let $R_{2}=\sigma_{n} Q_{2}$; then

$$
\xi=Q_{1} \operatorname{diag}\left(\epsilon_{1}, \cdots, \epsilon_{n-1}, 1\right) R_{2},
$$

where $\epsilon_{i}=\frac{\sigma_{i}}{\sigma_{n}} \in[-1,1]$ for $i=1, \cdots, n-1$. Let, for $j=0,1, \cdots, n-1$, $S_{j}=\left\{\operatorname{diag}\left(\epsilon_{1}, \cdots, \epsilon_{n}\right)|| \epsilon_{i}|\leq 1, \forall i \geq 1 ;| \epsilon_{k} \mid=1, \forall k \geq j+1 ; \epsilon_{n}=1\right\}$. (4.6)

Note that any $\eta=\operatorname{diag}\left(\epsilon_{1}, \cdots, \epsilon_{n}\right) \in S_{j}$ can be written as $\eta=t \eta^{+}+(1-$ t) $\eta^{-}$with $t=\left(1+\epsilon_{j}\right) / 2 \in[0,1]$ and

$$
\eta^{ \pm}=\operatorname{diag}\left(\epsilon_{1}, \cdots, \epsilon_{j-1}, \pm 1, \epsilon_{j+1}, \cdots, \epsilon_{n}\right) \in S_{j-1} ;
$$


clearly, $\operatorname{rank}\left(\eta^{+}-\eta^{-}\right)=1$. Hence, $S_{j} \subseteq \mathcal{L}_{1}\left(S_{j-1}\right)$ for $j=1,2, \cdots, n-1$. This proves $S_{n-1} \subseteq \mathcal{L}_{n-1}\left(S_{0}\right)$. Finally, by (4.5), we have

$$
\xi \in Q_{1} S_{n-1} R_{2} \subseteq Q_{1} \mathcal{L}_{n-1}\left(S_{0}\right) R_{2} \subseteq \mathcal{L}_{n-1}\left(Q_{1} S_{0} R_{2}\right) \subseteq \mathcal{L}_{n-1}\left(A_{\lambda}\right) .
$$

Therefore $B_{\lambda} \subseteq \mathcal{L}_{n-1}\left(A_{\lambda}\right)$; the proof is completed.

Proposition 4.2. Let $n \geq 3, L \geq 1$ and $1 \leq p<\frac{n L}{L+1}$, and let $\mathbf{B}$ be the open unit ball in $\mathbf{R}^{n}$. Then for any $\xi \in R(n)$ there exists a weakly $L$-quasiregular map $u_{\xi} \in \xi x+W_{0}^{1, p}\left(\mathbf{B} ; \mathbf{R}^{n}\right)$ such that

$$
\frac{1}{|\mathbf{B}|} \int_{\mathbf{B}}\left|D u_{\xi}\right|^{p} d x \leq C|\xi|^{p},
$$

where $C$ is a constant depending only on $n, L$ and $p$.

Proof. Let $\xi \in R(n)$ be given. If $\xi \in K_{1}$ we choose $u_{\xi} \equiv \xi x$. Assume $\xi \in R(n) \backslash K_{1}$. Define

$$
u_{\xi}(x)=\frac{\xi x}{|x|^{\delta}}, \quad \delta=\frac{L+1}{L}, \quad \forall x \neq 0 .
$$

Since $1 \leq p<\frac{n L}{L+1}$, an elementary computation shows that $u_{\xi} \in W^{1, p}(\mathbf{B}$; $\left.\mathbf{R}^{n}\right), D u_{\xi}(x) \in K_{L}$ (in fact $D u_{\xi}(x) \in \partial K_{L}$ ) for all $x \neq 0$ and $\left.u_{\xi}\right|_{\partial \mathbf{B}}=\xi x$. Furthermore, using the formula $\left|D u_{\xi}(x)\right|=|x|^{-\delta}|\xi|$, one easily has

$$
\frac{1}{|\mathbf{B}|} \int_{\mathbf{B}}\left|D u_{\xi}\right|^{p} d x \leq c_{n, p, \delta}|\xi|^{p} .
$$

This completes the proof. Finally let us notice that the maps $u_{\xi}$ we have used in the proof also satisfy $D u_{\xi}(x) \in K_{1} \cup \partial K_{L}$ for a.e. $x \in \mathbf{B}$.

\section{Proof of Theorem 1.3}

Let $\xi \in \mathbf{M}^{n \times n}$ be given. We assume $\xi \neq 0$. Let $\lambda=2|\xi|$. Then $\xi \in B_{\lambda}$. From Proposition 4.1, $\mathcal{L}\left(A_{\lambda}\right)=B_{\lambda}$ is open and bounded; one also has $\sup _{\eta \in B_{\lambda}}|\eta|^{p} \leq C_{1}|\xi|^{p}$. Also, from Proposition 4.2, the set $A=A_{\lambda} \subset$ $\beta_{p}\left(K_{L}\right)$ satisfies the condition (3.3) in Theorem 3.2 with constant $c_{0} \leq$ $C_{2}|\xi|^{p}$. Therefore, Theorem 3.2 implies $\xi \in \beta_{p}\left(K_{L}\right)$. This proves the theorem.

Using Lemma 3.1 and the remark following the proof of Theorem 3.2, we easily obtain the following result from Theorem 1.3.

Corollary 4.3. Let $n \geq 3, L \geq 1$ and $1 \leq p<\frac{n L}{L+1}$. Then, for any $\xi \in$ $\mathbf{M}^{n \times n}, b \in \mathbf{R}^{n}$ and $\epsilon>0$, there exists a weakly L-quasiregular map $u_{\epsilon} \in \xi x+b+W_{0}^{1, p}\left(\Omega ; \mathbf{R}^{n}\right)$ satisfying

$$
\int_{\Omega}\left|D u_{\epsilon}\right|^{p} d x \leq C_{3}|\xi|^{p}|\Omega|, \quad\left\|u_{\epsilon}-\xi x-b\right\|_{L^{p}(\Omega)}<\epsilon,
$$

where $C_{3}$ is a constant depending only on $n$ and $p$. 


\section{Proof of Theorem 1.5}

Let $n \geq 3, L \geq 1$ and $1 \leq p<\frac{n L}{L+1}$. Let $\varphi \in W^{1, p}\left(\Omega ; \mathbf{R}^{n}\right)$ be a piece-wise affine map. Let $\Omega=\cup_{j=1}^{\infty} \Omega_{j} \cup N,|N|=0$, be such that $\left.\varphi\right|_{\Omega_{j}}=\xi_{j} x+b_{j}$ is affine for all $j \geq 1$. The condition $\varphi \in W^{1, p}\left(\Omega ; \mathbf{R}^{n}\right)$ implies

$$
\sum_{j=1}^{\infty}\left|\xi_{j}\right|^{p}\left|\Omega_{j}\right|<\infty .
$$

We apply Corollary 4.3 to each $\xi_{j}, b_{j}$ and $\Omega_{j}$ and obtain weakly $L$-quasiregular maps $u_{j} \in \varphi+W_{0}^{1, p}\left(\Omega_{j} ; \mathbf{R}^{n}\right)$ satisfying

$$
\int_{\Omega_{j}}\left|D u_{j}\right|^{p} d x \leq C_{3}\left|\xi_{j}\right|^{p}\left|\Omega_{j}\right|, \quad\left\|u_{j}-\varphi\right\|_{L^{p}\left(\Omega_{j}\right)}<\frac{\epsilon}{2^{j / p}} .
$$

Then it is easily seen that the map $u=\sum_{j} \chi_{\Omega_{j}} u_{j}$ belongs to $\varphi+W_{0}^{1, p}(\Omega$; $\mathbf{R}^{n}$ ), is weakly $L$-quasiregular and satisfies $\|u-\varphi\|_{L^{p}(\Omega)}<\epsilon$. The proof is completed.

Finally, from the previous proofs and the note in the proof of Proposition 4.2 , we easily obtain the following slightly stronger result.

Theorem 4.4. Let $n \geq 3, L \geq 1$ and $1 \leq p<\frac{n L}{L+1}$. Then, for any piecewise affine map $\varphi \in W^{1, p}\left(\Omega ; \mathbf{R}^{n}\right)$ and $\epsilon>0$, there exists a map $u_{\epsilon} \in$ $\varphi+W_{0}^{1, p}\left(\Omega ; \mathbf{R}^{n}\right)$ such that $D u_{\epsilon}(x) \in K_{1} \cup \partial K_{L}$ a.e. in $\Omega$ and $\| u_{\epsilon}-$ $\varphi \|_{L^{p}(\Omega)}<\epsilon$.

Remark. We don't know whether the set $K_{1} \cup \partial K_{L}$ in this theorem can be replaced simply by $\partial K_{L}$.

Acknowledgement. The author would like to thank Tadeusz Iwaniec and Vladimír Šverák for their interest in the result of this paper. He also thanks Dr. M. A. Sychev for a careful reading of the original manuscript.

\section{References}

1. R. A. Adams, Sobolev Spaces, Academic Press, New York, 1975

2. K. Astala, Analytic aspects of quasiconformality, Proceedings of the International Congress of Mathematicians, Vol. II (Berlin, 1998). Doc. Math. 1998, Extra Vol. II, 617-626

3. J. M. Ball, Convexity conditions and existence theorems in nonlinear elasticity, Arch. Rational Mech. Anal. 63 (1977), 337-403

4. J. M. Ball and R. D. James, Proposed experimental tests of a theory of fine microstructures and the two well problem, Phil. Trans. Roy. Soc. London 338A (1992), 389-450

5. J. M. Ball and F. Murat, $W^{1, p}$-Quasiconvexity and variational problems for multiple integrals, J. Funct. Anal. 58 (1984), 225-253 
6. A. Cellina, On minima of a functional of the gradient: sufficient conditions, Nonlinear Anal. 20 (4) (1993), 343-347

7. B. Dacorogna, Direct Methods in the Calculus of Variations, Springer-Verlag, New York, 1989

8. B. Dacorogna and P. Marcellini, General existence theorems for Hamilton-Jacobi equations in the scalar and vectorial cases, Acta Math. 178 (1) (1997), 1-37

9. B. Dacorogna and P. Marcellini, Cauchy-Dirichlet problem for first order nonlinear systems, J. Funct. Anal. 152 (2) (1998), 404-446

10. M. Gromov, Partial Differential Relations, Springer-Verlag, New York, 1986

11. T. Iwaniec, $p$-Harmonic tensors and quasiregular mappings, Ann. of Math. (2) 136(3) (1992), 589-624

12. T. Iwaniec, Nonlinear Cauchy-Riemann operators in $\mathbf{R}^{n}$, Trans. Amer. Math. Soc., to appear

13. T. Iwaniec and A. Lutoborski, Integral estimates for null Lagrangians, Arch. Rational Mech. Anal. 125 (1993), 25-79

14. T. Iwaniec and G. Martin, Quasiregular mappings in even dimensions, Acta Math. 170 (1993), 29-81

15. T. Iwaniec and C. Sbordone, Weak minima of variational integrals, J. reine angew. Math. 454 (1994), 143-161

16. C. B. Morrey, Quasiconvexity and the lower semicontinuity of multiple integrals, Pacific J. Math. 2 (1952), 25-53

17. C. B. Morrey, Multiple Integrals in the Calculus of Variations, Springer-Verlag, Berlin, 1966

18. S. Müller and V. Šverák, Attainment results for the two-well problem by convex integration, in Geometric Analysis and the Calculus of Variations, pp. 239-251, Internat. Press, Cambridge, MA, 1996

19. S. Müller and V. Šverák, Unexpected solutions of first and second order partial differential equations, Proceedings of the International Congress of Mathematicians, Vol. II (Berlin, 1998). Doc. Math. 1998, Extra Vol. II, 691-702

20. S. Müller, V. Šverák and B. Yan, Sharp stability results for almost conformal maps in even dimensions, J. Geom. Anal. 9(4) (1999), 671-681

21. S. Müller and M. A. Sychev, Optimal existence theorems for nonhomogeneous differential inclusions, Preprint

22. P. Pedregal, Laminates and microstructure, European J. Appl. Math. 4(2) (1993), 121149

23. Y. G. Reshetnyak, Space Mappings with Bounded Distortion, Transl. Math. Mono., Amer. Math. Soc., Vol. 73, 1989

24. S. Rickman, Quasiregular Mappings, Springer-Verlag, Berlin, 1993

25. R. T. Rockafellar, Convex Analysis, Princeton Univ. Press, Princeton, NJ, 1972

26. B. Yan, Remarks about $W^{1, p}$-stability of the conformal set in higher dimensions, Ann. Inst. H. Poincaré, Analyse Non Linéaire 13 (6) (1996), 691-705

27. B. Yan, On rank-one convex and polyconvex conformal energy functions with slow growth, Proc. Roy. Soc. Edinburgh 127A (1997), 651-663

28. B. Yan, Linear boundary values of weakly quasiregular mappings, C. R. Acad. Sci. Paris Ser. I Math. 331 (2000), 379-384

29. B. Yan and Z. Zhou, Stability of weakly almost conformal mappings, Proc. Amer. Math. Soc. 126 (1998), 481-489

30. B. Yan and Z. Zhou, $L^{p}$-mean coercivity, regularity and relaxation in the calculus of variations, Nonlinear Anal., to appear

31. K. Zhang, On various semiconvex hulls in the calculus of variations, Calc. Var. Partial Differential Equations 6 (1998), 143-160 